\title{
A PALEY-WIENER THEOREM FOR FRAMES
}

\author{
OLE CHRISTENSEN
}

(Communicated by Palle E. T. Jorgensen)

\begin{abstract}
We prove a stability theorem for frames. Our result is a generalization of a classical result of Paley and Wiener about Riesz bases; it is also related to the Perturbation Theorem of Kato.
\end{abstract}

The classical Paley-Wiener Theorem states the following: Let $\left\{f_{i}\right\}_{i=1}^{\infty}$ be a basis for the Banach space $B$, and let $\left\{g_{i}\right\}_{i=1}^{\infty}$ be a family of vectors in $B$. If there exists a constant $\lambda \in[0 ; 1[$ such that

$$
\left\|\sum_{i=1}^{n} c_{i}\left(f_{i}-g_{i}\right)\right\| \leq \lambda \cdot\left\|\sum_{i=1}^{n} c_{i} f_{i}\right\|
$$

for all scalars $c_{1}, \ldots, c_{n}(n=1,2 \ldots)$, then $\left\{g_{i}\right\}_{i=1}^{\infty}$ is a basis for $B$.

Intuitively, the statement is that any family which is sufficiently close to a basis (in the sense above) is a basis. The proof is not difficult. The conditions imply that there exists a bounded invertible operator $T$ such that $T f_{i}=g_{i}$.

The above formulation is due to Boas (cf. [Y]). The Paley-Wiener Theorem is useful in order to show that a family $\left\{g_{i}\right\}_{i=1}^{\infty}$ is a Riesz basis for a Hilbert space, so the result is sometimes used in wavelet analysis ([B], [S]). But in many cases the wavelet experts prefer to work with frames instead of Riesz bases; our aim here is to show that a similar result holds for frames, however with a completely different proof.

The needed facts about frames can be found in the paper [C].

Theorem 1. Let $\mathscr{H}$ be a Hilbert space and $\left\{f_{i}\right\}_{i=1}^{\infty}$ a frame for $\mathscr{H}$ with bounds $A$ and $B$. Let $\left\{g_{i}\right\}_{i=1}^{\infty}$ be a family of elements in $\mathscr{H}$, and suppose that

$\exists \lambda, \mu \geq 0: \lambda+\frac{\mu}{\sqrt{A}}<1$ and $\left\|\sum_{i=1}^{n} c_{i}\left(f_{i}-g_{i}\right)\right\| \leq \lambda \cdot\left\|\sum_{i=1}^{n} c_{i} f_{i}\right\|+\mu \cdot\left[\sum_{i=1}^{n}\left|c_{i}\right|^{2}\right]^{1 / 2}$

for all $c_{1}, \ldots, c_{n}(n=1,2, \ldots)$. Then $\left\{g_{i}\right\}_{i=1}^{\infty}$ is a frame with bounds $A(1-(\lambda+\mu / \sqrt{A}))^{2}, B(1+\lambda+\mu / \sqrt{B})^{2}$.

Received by the editors October 4, 1993 and, in revised form, November 17, 1993.

1991 Mathematics Subject Classification. Primary 42A99, 42C15.

The author would like to thank the Carlsberg Foundation and the Danish Research Council for financial support.

(C)1995 American Mathematical Society 
Proof. Consider the operator

$$
U: l^{2}(\mathbf{N}) \rightarrow \mathscr{H}, \quad U\left\{c_{i}\right\}:=\sum_{i=1}^{\infty} c_{i} f_{i} .
$$

Frame theory says that $U$ is well defined, bounded, and that $\|U\| \leq \sqrt{B}$. The assumptions imply that we can define an operator

$$
T: l^{2}(\mathbf{N}) \rightarrow \mathscr{H}, \quad T\left\{c_{i}\right\}:=\sum_{i=1}^{\infty} c_{i} g_{i}
$$

and that

$$
\left\|U\left\{c_{i}\right\}-T\left\{c_{i}\right\}\right\| \leq \lambda \cdot\left\|U\left\{c_{i}\right\}\right\|+\mu \cdot\left\|\left\{c_{i}\right\}\right\|, \quad \forall\left\{c_{i}\right\} \in l^{2}(\mathbf{N}) .
$$

Therefore

$$
\left\|T\left\{c_{i}\right\}\right\| \leq[(1+\lambda) \sqrt{B}+\mu] \cdot\left\|\left\{c_{i}\right\}\right\|, \quad \forall\left\{c_{i}\right\} \in l^{2}(\mathbf{N}) .
$$

That is, $\left\{g_{i}\right\}_{i=1}^{\infty}$ is a Bessel sequence with upper bound $[\sqrt{B}(\lambda+1)+\mu]^{2}=$ $B(1+\lambda+\mu / \sqrt{B})^{2}$.

Now we verify the existence of the lower frame bound for $\left\{g_{i}\right\}_{i=1}^{\infty}$. Observe that $U U^{*}$ is the frame operator for $\left\{f_{i}\right\}_{i=1}^{\infty}$ and therefore invertible. Let us consider the operator

$$
U^{\dagger}: \mathscr{H} \rightarrow l^{2}(\mathbf{N}), \quad U^{\dagger} f:=U^{*}\left(U U^{*}\right)^{-1} f=\left\{\left\langle f,\left(U U^{*}\right)^{-1} f_{i}\right\rangle\right\} .
$$

$\left\{\left(U U^{*}\right)^{-1} f_{i}\right\}_{i=1}^{\infty}$ is a frame with upper bound $1 / A$, so

$$
\left\|U^{\dagger} f\right\|^{2}=\sum_{i=1}^{\infty}\left|\left\langle f,\left(U U^{*}\right)^{-1} f_{i}\right\rangle\right|^{2} \leq \frac{1}{A} \cdot\|f\|^{2}, \quad \forall f \in \mathscr{H} .
$$

Using (1) with $\left\{c_{i}\right\}=U^{\dagger} f$ we get

$$
\left\|f-T U^{\dagger} f\right\| \leq\left(\lambda+\frac{u}{\sqrt{A}}\right) \cdot\|f\|, \quad \forall f \in \mathscr{H} .
$$

Therefore $T U^{\dagger}$ is invertible, and

$$
\left\|T U^{\dagger}\right\| \leq 1+\lambda+\frac{\mu}{\sqrt{A}}, \quad\left\|\left(T U^{\dagger}\right)^{-1}\right\| \leq 1 /(1-(\lambda+\mu / \sqrt{A})) .
$$

Any $f \in \mathscr{H}$ can be written as

$$
f=T U^{\dagger}\left(T U^{\dagger}\right)^{-1} f=\sum_{i=1}^{\infty}\left\langle\left(T U^{\dagger}\right)^{-1} f,\left(U U^{*}\right)^{-1} f_{i}\right\rangle g_{i} ;
$$

thus

$$
\begin{aligned}
\|f\|^{4} & =\langle f, f\rangle^{2}=\left|\sum_{i=1}^{\infty}\left\langle\left(T U^{\dagger}\right)^{-1} f,\left(U U^{*}\right)^{-1} f_{i}\right\rangle\left\langle g_{i}, f\right\rangle\right|^{2} \\
& \leq\left.\sum_{i=1}^{\infty}\left|\left(T U^{\dagger}\right)^{-1} f,\left(U U^{*}\right)^{-1} f_{i}\right\rangle\right|^{2} \cdot \sum_{i=1}^{\infty}\left|\left\langle g_{i}, f\right\rangle\right|^{2} \\
& \leq \frac{1}{A} \cdot\left\|\left(T U^{\dagger}\right)^{-1} f\right\|^{2} \cdot \sum_{i=1}^{\infty}\left|\left\langle g_{i}, f\right\rangle\right|^{2} \\
& \leq \frac{1}{A\left(1-\left(\lambda+\frac{\mu}{\sqrt{A}}\right)\right)^{2}} \cdot\|f\|^{2} \cdot \sum_{i=1}^{\infty}\left|\left\langle g_{i}, f\right\rangle\right|^{2}, \quad \forall f \in \mathscr{H} .
\end{aligned}
$$


Remarks. (1) Suppose that $\left\{f_{i}\right\}_{i=1}^{\infty}$ is a frame with bounds $A, B$ and that $\left\{g_{i}\right\}_{i=1}^{\infty}$ is any family such that $R:=\sum_{i=1}^{\infty}\left\|f_{i}-g_{i}\right\|^{2}<A$. Then the condition in Theorem 1 is satisfied with $\lambda=0$ and $\mu=\sqrt{R}$. Thus Theorem 1 generalizes Theorem 1 in [C].

(2) The condition in Theorem 1 implies that $\lambda<1$. This is essential. Let $\left\{f_{i}\right\}_{i=1}^{\infty}$ be an orthonormal basis for $\mathscr{H}$, and define $g_{i}:=f_{i}+f_{i+1}$. Then $\overline{\operatorname{span}}\left\{g_{i}\right\}=\mathscr{H}$, but $\left\{g_{i}\right\}$ is not a frame;

$$
\sum\left|\left\langle f, g_{i}\right\rangle\right|^{2}=1 / n \cdot\|f\|^{2}
$$

with $f:=\sum_{i=1}^{n}(-1)^{j-1} e_{j}$, so the lower frame condition is not satisfied. Since

$$
\left\|\sum_{i=1}^{n} c_{i}\left(f_{i}-g_{i}\right)\right\|=\left\|\sum_{i=1}^{n} c_{i} f_{i+1}\right\|=\left\|\sum_{i=1}^{n} c_{i} f_{i}\right\| \text {, }
$$

the example corresponds to $\lambda=1, \mu=0$.

(3) Our result is connected with the work of Kato (e.g., [K], p. 190). Consider the operator $T$ as a perturbation of $U$; in the terminology of Kato, the condition in Theorem 1 implies that the "perturbation operator" $T-U$ is $U$-bounded with $U$-bound smaller than 1 .

(4) In the classical Paley-Wiener Theorem, the conditions imply that $\sum_{i=1}^{n} c_{i} g_{i}=0$ if and only if $\sum_{i=1}^{n} c_{i} f_{i}=0$. So the sets $\left\{f_{i}\right\}$ and $\left\{g_{i}\right\}$ must have the same linear dependence. This is automatically satisfied if $\left\{f_{i}\right\}$ and $\left\{g_{i}\right\}$ are bases, but in general it is a strong condition. We have avoided this obstacle in Theorem 1; from this point of view the introduction of $\mu$ plays an important role.

(5) Theorem 1 has applications to the important coherent frames, shortly discussed in [C]. For example, the proof of Theorem 5 in [S] uses the PaleyWiener Theorem; it can be expected that a similar result can be proved for frames, using Theorem 1. Also, our result is applicable to the important problem of perturbation of the mother wavelet $f$ in a coherent frame $\left\{\pi\left(x_{i}\right) f\right\}_{i=1}^{\infty}$. We refer to the paper [FZ], where the reader also finds other applications of Theorem 1.

\section{ACKNOWLEDGMENT}

The author would like to thank Erik Balslev and Ebbe Thue Poulsen for discussions leading to the final version of the main result.

\section{REFERENCES}

[B] J. J. Benedetto, Irregular sampling and frames, Wavelets-A Tutorial in Theory and Applications (C. K. Chui, ed.), Academic Press, New York, 1992.

[C] O. Christensen, Frame perturbation, Proc. Amer. Math. Soc. 123 (1995), 1217-1220.

[FZ] S. J. Favier and R. Zalik, On the stability of frames and Riesz bases (submitted).

[K] T. Kato, Perturbation theory for linear operators, Springer, New York, 1976.

[S] K. Seip, Sampling, interpolation and phase space density, Wavelets-A Tutorial in Theory and Applications (C. K. Chui, ed.), Academic Press, New York, 1992.

[Y] R. Young, Non-harmonic Fourier series, Academic Press, New York, 1980.

Matematisk Institut, Danmarks Tekniske Universitet, Bygning 303, 2800 Lyngby, DenMARK

E-mail address: OLECHROMAT.DTU.DK 